\title{
Minimum cost design of a cellular plate loaded by uniaxial compression
}

Károly Jármai, József Farkas

University of Miskolc, H-3515 Miskolc, Egyetemváros, Hungary

altjar@uni-miskolc.hu, altfar@uni-miskolc.hu

\begin{abstract}
Cellular plates are constructed from two base plates and an orthogonal grid of stiffeners welded between them. Halved rolled I-section stiffeners are used for fabrication aspects. The torsional stiffness of cells makes the plate very stiff. In the case of uniaxial compression the buckling constraint is formulated on the basis of the classic critical stress derived from the Huber's equation for orthotropic plates. The cost function contains the cost of material, assembly and welding and is formulated according to the fabrication sequence. The unknown variables are the base plate thicknesses, height of stiffeners and numbers of stiffeners in both directions. The cellular plate is lighter and cheaper than the plate stiffened on one side. The Particle Swarm Optimization and the IOSO techniques are used to find the optimum. PSO contains crazy bird and dynamic inertia reduction criteria, IOSO is based on a response surface technology.
\end{abstract}

Keywords: cellular plates, stiffened plates, cost calculation, structural optimization, welded structures, buckling strength

\section{Introduction}

Cellular plates can be applied in various structures e.g. in floors and roofs of buildings, in bridges, ships, machine structures etc. Cellular plates have the following advantages over the plates stiffened on one side: (a) because of their large torsional stiffness the plate thickness can be decreased, which results in decrease of welding cost, (b) their planar surface is more suitable to corrosion protection, (c) their quasi-symmetric welds do not cause residual distortion.

In previous studies (Farkas 1985, Farkas \& Jármai 2006) it has been shown that cellular plates can be calculated as isotropic ones, bending moments and deflections can be determined by using classic results of isotropic plates for various load and support types. 
A large research project was performed by Williams (1969) who used a welded cellular plate model for double bottom of ships. Pettersen (1979) has worked out a detailed analysis of double-bottom plates of ships. Evans and Shanmugam (1984), Shanmugam and Evans (1984) and Shanmugam and Balendra (1986) have treated the analytical problems of cellular plates relating to the ship construction.

A base plate for transportation of heavy structures may be built by using an orthogonal grid welded from rolled I-beams. The lower face plate has been joined to the grid by plug welds Sahmel (1978). In the revolving frame of surface mining equipment (dragline) a platform for boom, cab, power unit and other structural parts forms an all-welded multi-cell structure Birchfield (1981). Laser welding technology has been used for welding of "Norsial" metallic sandwich plates and a corrugated sheet sandwiched between them (Haroutel 1982).

In the book Farkas \& Jármai (1997) some problems can be found about cellular plates. Welded cellular plates for ships investigated in (Farkas \& Jármai 1999, 2003) consist of two face sheets and some longitudinal ribs of square hollow section welded between them using arc-spot welding technology. For stiffened plates we have investigated both the uniaxial and biaxial compression and bending.

In the present study the load is uniaxial compression, the stiffening is constructed with longitudinal halved rolled I-section stiffeners, the material is a higherstrength steel with yield stress of $355 \mathrm{MPa}$, the fabrication technology is welding (continuous longitudinal fillet submerged arc - SAW- welds). Our another study related to cellular plate in bending can be found in Farkas \& Jármai 2008. Further study is needed to consider combined loading, compression and bending.

We have developed a cost calculation method mainly for welded structures (Farkas \& Jármai 1997, 1999, 2003, 2008), by which it is possible to give a 
realistic cost comparison of optimized structural versions. The cost function includes the costs of material, assembly, welding, post-welding works and painting.

\section{The basic formulae of cellular plates}

The Huber's equation for the deflection $w(x, y)$ orthotropic plates in the case of a uniaxial compression $N_{x}$

$B_{x} \frac{\partial^{4} w}{\partial x^{4}}+2 H \frac{\partial^{4} w}{\partial x^{2} \partial y^{2}}+B_{y} \frac{\partial^{4} w}{\partial y^{4}}+N_{x} \frac{\partial^{2} w}{\partial x^{2}}=0$

where

$$
H=B_{x y}+B_{y x}+\frac{v}{2}\left(B_{x}+B_{y}\right)
$$

is the torsional stiffness of an orthotropic plate, $v=0.3$ is the Poisson's ratio.

The corresponding bending and torsional stiffnesses are defined as

$B_{x}=\frac{E_{1} I_{y}}{a_{y}} ; B_{y}=\frac{E_{1} I_{x}}{a_{x}} ; E_{1}=\frac{E}{1-v^{2}}$

$E=2.1 \times 10^{5} \mathrm{MPa}$ is the elastic modulus.

For cellular plates with the shear modulus $G$

$$
\begin{aligned}
& B_{x y}=\frac{G I_{y}}{a_{y}} ; B_{y x}=\frac{G I_{x}}{a_{x}} ; G=\frac{E}{2(1+v)} \\
& H=B_{x y}+B_{y x}+\frac{v}{2}\left(B_{x}+B_{y}\right)=\frac{E_{1}}{2}\left(\frac{I_{y}}{a_{y}}+\frac{I_{x}}{a_{x}}\right)
\end{aligned}
$$

The solution of Eq. (1) is given by

$$
N_{E}=\frac{\pi^{2}}{b_{0}^{2}}\left[B_{x}\left(\frac{b_{0}}{a_{0}}\right)^{2}+2 H+B_{y}\left(\frac{a_{0}}{b_{0}}\right)^{2}\right]
$$

\section{The overall buckling constraint}

The buckling constraint is given by 
$\frac{N_{x}}{n_{y} A_{e y}} \leq \sigma_{c r}=\frac{f_{y}}{\sqrt{1+\lambda^{4}}}, \lambda=\sqrt{\frac{f_{y}}{\sigma_{E}}}, \sigma_{E}=\frac{N_{E} s_{y}}{A_{e y}}$

The classic critical buckling stress $\sigma_{E}$ should be decreased using the above formulae, since it does not include the effect of initial imperfections and residual welding stresses. The DNV design rules are used for this decreasing (DNV 1995). $f_{y}$ is the yield stress.
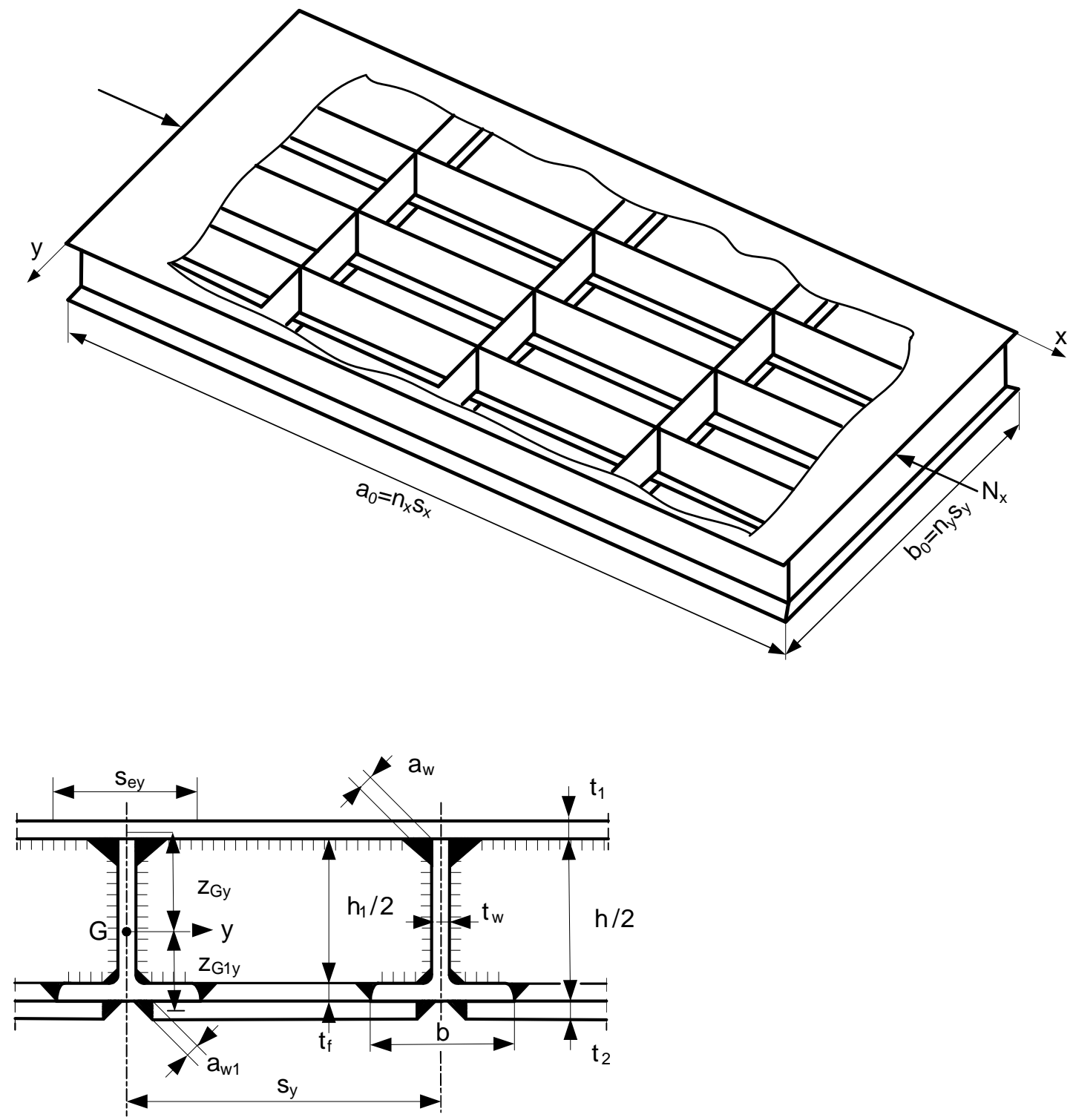

Figure 1. Orthogonally stiffened cellular plate and its cross-section

$A_{e y}=\frac{h_{1} t_{w}}{2}+b t_{f}+s_{e y 1} t_{1}+s_{e y 2} t_{2}$ 


$$
\begin{aligned}
& A_{e x}=\frac{h_{1} t_{w}}{2}+b t_{f}+s_{e x 1} t_{1}+s_{e x 2} t_{2} \\
& h_{1}=h-2 t_{f} \\
& s_{y}=\frac{b_{0}}{n_{y}}, s_{x}=\frac{a_{0}}{n_{x}}
\end{aligned}
$$

where $n_{y}$ and $n_{x}$ are the spacings of stiffeners in $y$ and $x$ directions (Fig.1).

The effective widths of plate parts can be calculated according to Eurocode 3 Part $1-5$ (2006) as

$S_{e y 1}=\rho_{y 1} s_{y}, s_{e y 2}=\rho_{y 2} s_{y}, s_{e x 1}=\rho_{x 1} s_{x}, s_{e x 2}=\rho_{x 2} s_{x}$

where

$$
\begin{array}{llll}
\rho_{y 1}=\frac{\lambda_{p y 1}-0.22}{\lambda_{p y 1}^{2}} & \text { if } & \lambda_{p y 1}=\frac{s_{y}}{56.8 \varepsilon t_{1}} \geq 0.673, \varepsilon=\sqrt{\frac{235}{f_{y}}} \\
\rho_{y 1}=1 & & \text { if } \quad \lambda_{p y 1}<0.673 \\
\rho_{y 2}=\frac{\lambda_{p y 2}-0.22}{\lambda_{p y 2}^{2}} & \text { if } & \lambda_{p y 2}=\frac{s_{y}}{56.8 \varepsilon t_{1}} \geq 0.673 \\
\rho_{y 2}=1 & \text { if } & \lambda_{p y 2}<0.673 \\
\rho_{x 1}=\frac{\lambda_{p x 1}-0.22}{\lambda_{p x 1}^{2}} & \text { if } & \lambda_{p x 1}=\frac{s_{x}}{56.8 \varepsilon t_{2}} \geq 0.673 \\
\rho_{x 1}=1 & \text { if } & \lambda_{p x 1}<0.673 \\
\rho_{x 2}=\frac{\lambda_{p x 2}-0.22}{\lambda_{p x 2}^{2}} & \text { if } & \lambda_{p x 2}=\frac{s_{x}}{56.8 \varepsilon t_{2}} \geq 0.673 \\
\rho_{x 2}=1 & \text { if } & \lambda_{p x 2}<0.673
\end{array}
$$

The distances of the gravity centres are expressed as

$$
z_{G y}=\frac{1}{A_{e y}}\left[\frac{h_{1} t_{w}}{2}\left(\frac{h_{1}}{4}+\frac{t_{1}}{2}\right)+b t_{f}\left(\frac{h_{1}+t_{f}+t_{1}}{2}\right)+s_{e y 2} t_{2}\left(\frac{h_{1}+t_{f}+t_{1}+t_{2}}{2}\right)\right]
$$




$$
z_{G x}=\frac{1}{A_{e x}}\left[\frac{h_{1} t_{w}}{2}\left(\frac{h_{1}}{4}+\frac{t_{1}}{2}\right)+b t_{f}\left(\frac{h_{1}+t_{f}+t_{1}}{2}\right)+s_{e x 2} t_{2}\left(\frac{h_{1}+t_{f}+t_{1}+t_{2}}{2}\right)\right]
$$

and the moments of inertia are given by

$$
\begin{aligned}
& I_{y}=s_{e y 1} t_{1} z_{G y}^{2}+\frac{h_{1}^{3} t_{w}}{96}+\frac{h_{1} t_{w}}{2}\left(\frac{h_{1}}{4}+\frac{t_{1}}{2}-z_{G y}\right)^{2}+I_{y 1} \\
& I_{y 1}=b t_{f}\left(\frac{h_{1}+t_{f}+t_{1}}{2}-z_{G y}\right)^{2}+s_{e y 2} t_{2}\left(\frac{h_{1}+t_{f}+t_{1}+t_{2}}{2}-z_{G y}\right)^{2}
\end{aligned}
$$

$I_{x}=s_{e x 1} t_{1} z_{G x}^{2}+\frac{h_{1}^{3} t_{w}}{96}+\frac{h_{1} t_{w}}{2}\left(\frac{h_{1}}{4}+\frac{t_{1}}{2}-z_{G x}\right)^{2}+I_{x 1}$

$I_{x 1}=b t_{f}\left(\frac{h_{1}+t_{f}+t_{1}}{2}-z_{G x}\right)^{2}+s_{e x 2} t_{2}\left(\frac{h_{1}+t_{f}+t_{1}+t_{2}}{2}-z_{G x}\right)^{2}$

The fabrication constraint makes it possible to weld the fillet welds connecting the web of the stiffeners to the upper base plate

$s_{y, x}-b \geq 300 m m$

The unknowns are as follows: $x_{1}=t_{1}$ upper cover plate thickness, $x_{2}=t_{2}$ lower cover plate thickness, $x_{3}=h$ height of the I beam, $x_{4}=n_{x}$ number of stiffeners in $\mathrm{x}$ directions, $x_{5}=n_{y}$ number of stiffeners in y-directions.

\section{The cost function}

There are relatively few papers using cost calculation (Krack et al. 2011, Pavlovcic et. al. 2004, Sarma and Adeli 2002, Kravanja et al. 2008). In this paper the cost consists of the cost of material $\left(K_{M}\right)$ and welding $\left(K_{W}\right)$.

$K_{M}=k_{M} \rho V$

where $k_{M}=1.0 \$ / \mathrm{kg}, \rho=7.85 \times 10^{-6} \mathrm{~kg} / \mathrm{mm}^{3}, V$ is the volume.

The general formula for the welding cost is as follows (Farkas \& Jármai 1997, $1999,2003,2008)$ 


$$
K_{w}=k_{w}\left(C_{1} \Theta \sqrt{\kappa \rho V}+1.3 \sum_{i} C_{w i} a_{w i}^{n} C_{p i} L_{w i}\right)
$$

where $k_{w}[\$ / \mathrm{min}]$ is the welding cost factor, $C_{1}$ is the factor for the assembly usually taken as $C_{l}=1 \mathrm{~min} / \mathrm{kg}^{0.5}, \Theta$ is the factor expressing the complexity of assembly, the first member calculates the time of the assembly, $\kappa$ is the number of structural parts to be assembled, $\rho V$ is the mass of the assembled structure, the second member estimates the time of welding, $C_{w}$ and $n$ are the constants given for the specified welding technology and weld type, $C_{p}$ is the factor of welding position (for downhand 1 , for vertical 2 , for overhead 3 ) $L_{w}$ is the weld length, the multiplier 1.3 takes into account the additional welding times (deslagging, chipping, changing the electrode).

The welding costs are formulated according to the fabrication sequence.

(a) Welding the upper base plate with SAW (submerged arc welding) butt welds. The weld length is $L_{W 1}=3\left(a_{0}+b_{0}\right)$, the structural volume $V_{1}=a_{0} b_{0} t_{1}$, $\Theta_{1}=2$, number of elements $\kappa_{1}=16 . k_{W}=1.0 \$ / \mathrm{min}$.

For $t_{1} \geq 15 \mathrm{~mm} C_{W} a_{W}^{n}=0.1346 \times 10^{-3} t_{1}^{2}$

and for $t_{1}<15 \mathrm{~mm} C_{W} a_{W}^{n}=0.1033 \times 10^{-3} t_{1}^{1.94}$

$$
K_{W 1}=k_{W}\left(\Theta_{1} \sqrt{\kappa_{1} \rho V_{1}}+1.3 C_{W} a_{W}^{n} L_{W 1}\right) .
$$

(b) Welding of longitudinal stiffeners to the upper base plate with two SAW fillet welds.

$$
\begin{aligned}
& L_{W 2}=2 a_{0}\left(n_{y}+1\right), \kappa_{2}=n_{y}+2, V_{2}=V_{1}+a_{0}\left(b t_{f}+h_{1} t_{w} / 2\right)\left(n_{y}+1\right), a_{W}=0.4 t_{w}, \\
& \Theta_{2}=3 . \\
& K_{W 2}=k_{W}\left(\Theta_{2} \sqrt{\kappa_{2} \rho V_{2}}+1.3 \times 0.2349 \times 10^{-3} a_{W}^{2} L_{W 2}\right) .
\end{aligned}
$$


(c) Welding of transverse stiffener parts to the upper base plate and to the longitudinal stiffeners, the webs with double fillet welds (GMAW-C gas metal arc welding with $\mathrm{CO}_{2}$ ) and flanges with butt welds.

$$
\begin{aligned}
& V_{3}=V_{2}+b_{0}\left(b t_{f}+h_{1} t_{w} / 2\right)\left(n_{x}+1\right), \\
& L_{W 3}=\left(n_{x}+1\right)\left(2 b_{0}+n_{y}\left(h_{1}+b\right)\right) \\
& K_{W 3}=k_{W}\left(\Theta_{2} \sqrt{\kappa_{3} \rho V_{3}}+1.3 x 0.3394 x 10^{-3} a_{W}^{2} L_{W 3}+1.3 C_{W f} t_{f}^{n} L_{W f}\right)
\end{aligned}
$$

For $t_{f} \geq 15 \mathrm{~mm} C_{W f} t_{f}^{n}=0.1496 \times 10^{-3} t_{f}^{1.9029}$,

for $t_{f}<15 \mathrm{~mm} \quad C_{W f} t_{f}^{n}=0.1939 \times 10^{-3} t_{f}^{2}$

$$
L_{W f}=2 b n_{y}\left(n_{x}+1\right)
$$

(d) Welding of lower base plate elements to the flanges of stiffeners with SAW fillet welds.

$$
\begin{aligned}
& V_{4}=V_{3}+a_{0} b_{0} t_{2}, \kappa_{4}=1+n_{x} n_{y}, L_{W 4}=2\left(a_{0} n_{y}+b_{0} n_{x}\right), a_{W 1}=0.7 t_{2}, \\
& K_{W 4}=k_{W}\left(\Theta_{1} \sqrt{\kappa_{4} \rho V_{4}}+1.3 \times 0.2349 \times 10^{-3} a_{W 1}^{2} L_{W 4}\right) .
\end{aligned}
$$

The total cost is

$$
K=K_{M}+K_{W 1}+K_{W 2}+K_{W 3}+K_{W 4} .
$$

\section{The Particle Swarm Optimization technique}

Several methods have been developed to escape from being caught in local optima. The Particle Swarm Method of global optimization is one of such methods. A swarm of birds searches for food, protection, etc. in a very typical manner. If one of the members of the swarm sees a desirable path to go, the rest of the swarm will follow quickly. Every member of the swarm searches for the best in its locality - learns from its own experience.

Additionally, each member learns from the others, typically from the best performer among them. Even human beings show a tendency to learn from their 
own experience, their immediate neighbours and the ideal performers. The Particle Swarm method of optimization mimics this behaviour Kennedy \& Eberhart (1995). Every individual of the swarm is considered as a particle in a multidimensional space that has a position and a velocity. These particles fly through hyperspace and remember the best position that they have seen. Members of a swarm communicate good positions to each other and adjust their own position and velocity based on these good positions. The Particle Swarm method of optimization testifies the success of bounded rationality and decentralized decision making in reaching at the global optima. It has been used successfully to optimize extremely difficult multimodal functions.

Each particle keeps track of its coordinates in the problem space which are associated with the best solution (fitness) it has achieved so far. (The fitness value is also stored.) This value is called pbest. Another "best" value that is tracked by the particle swarm optimizer is the best value, obtained so far by any particle in the neighbours of the particle. This location is called lbest. when a particle takes all the population as its topological neighbours, the best value is a global best and is called gbest.

The particle swarm optimization concept consists of, at each time step, changing the velocity of (accelerating) each particle toward its pbest and lbest locations (local version of $P S O$ ). Acceleration is weighted by a random term, with separate random numbers being generated for acceleration toward pbest and lbest locations.

Another reason that $P S O$ is attractive is that there are few parameters to adjust. One version, with slight variations, works well in a wide variety of applications. 
The method is derivative free, and by its very nature the method is able to locate the global optimum of an objective function. Constrained problems can simply be accommodated using penalty methods (Fourie and Groenwold 2000).

Lately, the PSO was successfully applied to the optimum shape and size design of structures by Fourie and Groenwold (2000). An operator, namely craziness, was re-introduced, together with the use of dynamic varying maximum velocities and inertia.

\section{The pseudo code of the procedure can be written as follows:}

I) For each particle:

Initialize particle

II) $\mathrm{Do}$ :

a) For each particle:

1) Calculate fitness value

2) If the fitness value is better than the best fitness value (pbest) in history

3) Set current value as the new pbest

\section{End}

b) For each particle:

1) Find in the particle neighbourhood, the particle with the best fitness

2) Calculate particle velocity according to the velocity equation (34)

3) Apply the velocity constriction

4) Update particle position according to the position equation (35)

5) Apply the position constriction

\section{End}

While maximum iterations or minimum error criteria is not attained. 
A more precise and detailed description of the particular PSO algorithm, as applied to penalty function formulation and used in this study now follows.

\section{Basic PSO Algorithm}

Given $M, k_{\max }, N_{\max }$. Set (time) instant $k=0, F_{i}^{b}=F^{g}=F_{\text {before }}^{g}=\infty$. Initialise a random population (swarm) of $M$ particles (swarm members), by assigning an initial random position $\boldsymbol{x}_{\mathrm{i}}^{0}$ (candidate solution), as well as a random initial velocity $\boldsymbol{v}_{i}^{o}$, to each particle $i, i=1,2, \ldots, M$. Then compute simultaneous trajectories, one for each particle, by performing the following steps.

1) At instant $k$, compute the fitness of each individual particle $i$ at discrete point $\boldsymbol{x}_{i}^{k}$, by evaluating $F\left(\boldsymbol{x}_{i}^{k}\right)$. With reference to the minimization of (1.4), the lower the value of $F\left(\boldsymbol{x}_{i}^{k}\right)$, the greater the particle's fitness.

2) For $i=1,2, \ldots, M$ :

if $F\left(\boldsymbol{x}_{i}^{k}\right) \leq F_{i}^{b}$ then set $F_{i}^{b}=F\left(\boldsymbol{x}_{i}^{k}\right)$ and $\boldsymbol{p}_{i}^{b}=\boldsymbol{x}_{i}^{k} \quad$ \{best point on trajectory $\left.i\right\}$

if $F\left(x_{i}^{k}\right) \leq F^{g}$ then set $F^{g}=F\left(x_{i}^{k}\right)$ and $\boldsymbol{g}^{b}=\boldsymbol{x}_{i}^{k}$ \{best global point $\}$

3) If $F^{g}<F_{\text {before }}^{g}$ then set $N=1$, else set $N=N+1$.

4) If $N>N_{\max }$ or $k>k_{\max }$ then STOP and set $\boldsymbol{x}^{*}=\boldsymbol{g}^{b}$; else continue.

5) Compute new velocities and positions for instant $k+1$, using the rule:

$$
\begin{aligned}
& \text { for } i=1,2, \ldots, \mathrm{M}: \\
& \boldsymbol{v}_{i}^{k+1}:=\boldsymbol{v}_{i}^{k}+c_{1} r_{1}\left(\boldsymbol{p}_{i}^{b}-\boldsymbol{x}_{i}^{k}\right)+c_{2} r_{2}\left(\boldsymbol{g}^{b}-\boldsymbol{x}_{i}^{k}\right), \\
& \boldsymbol{x}_{i}^{k+1}:=\boldsymbol{x}_{i}^{k}+\boldsymbol{v}_{i}^{k+1} t,
\end{aligned}
$$

where $r_{1}$ and $r_{2}$ are independently generated random numbers in the interval $[0,1]$, and $c_{1}, c_{2}$ are parameters with appropriately chosen values. 
6) Set $k=k+1$ and $F_{\text {before }}^{g}=F^{g}$; go to step 2 .

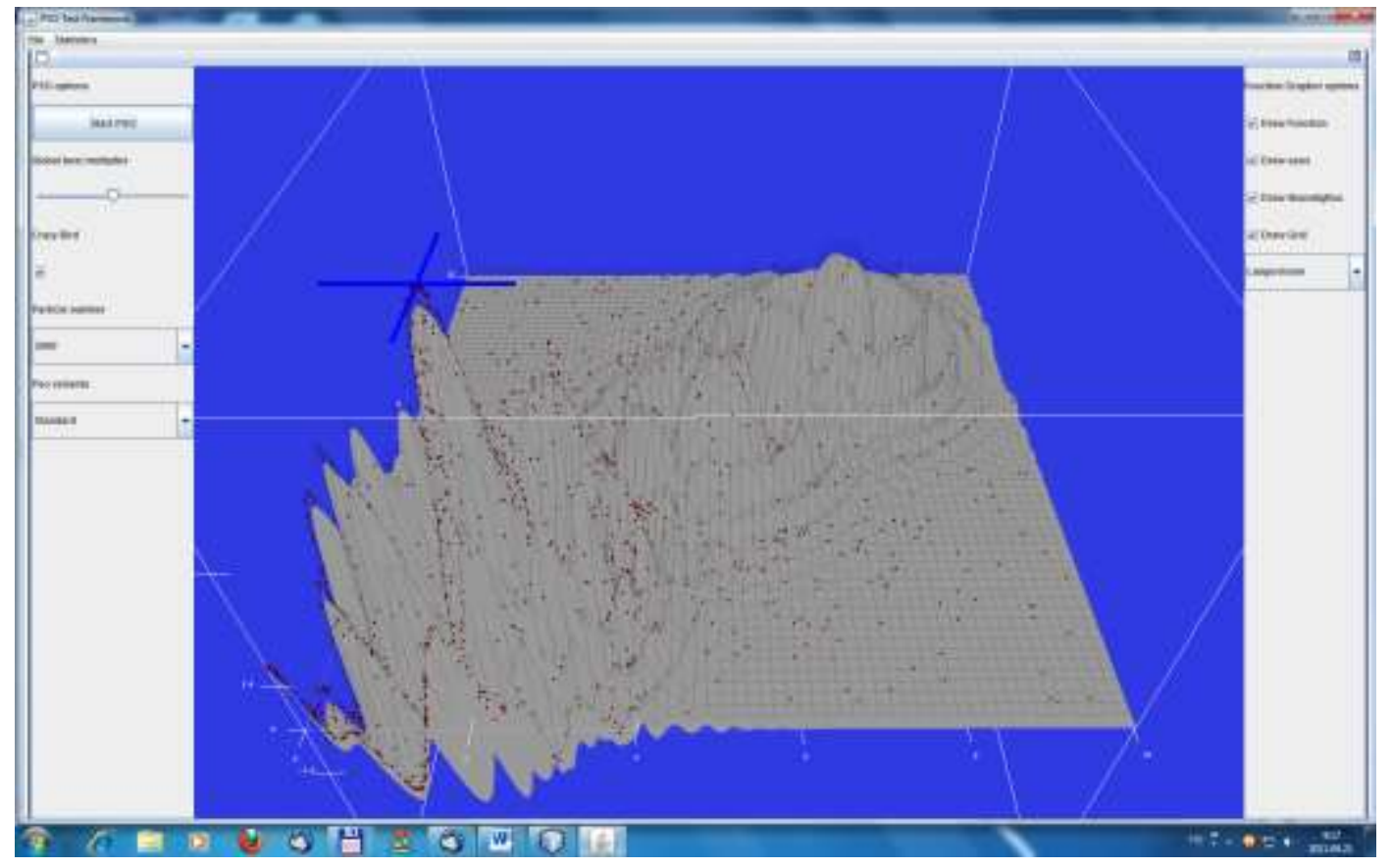

Figure 2. PSO with 2000 particles in action for a test function

The technique is modified in order to be efficient in technical applications. It uses dynamic inertia reduction and craziness for some particles (Fourie \& Groenwold 2000). Figure 2 shows the PSO in action, solving a test problem with 2000 particles, introducing crazy bird operation.

\section{The IOSO technique}

\section{Main features of IOSO technology}

IOSO Technology (Indirect Optimization on Self-Organization) is based on the response surface technology. That is why our strategy differs significantly from the well-known approaches to optimization. Our strategy has higher efficiency and provides wider range of capabilities than standard algorithms. The main advantage one can get from using the IOSO Technology is ability to solve very complex optimization tasks. 
There are several response surface technology algorithms. Some of them are very attractive (Xiong 2011). IOSO algorithms are specifically developed for solving optimization problems. They are accurate in predicting the direction towards the optimum. We can approximate objective functions with complex topology (including the ones with local optima) using minimal number of points in the experiment plan, particularly including the case when the number of points is less than the number of design variables. For example, we start solution of the optimization problem with 140 design variables using only 40 points.

Each iteration of IOSO algorithm consists of two steps. The first step is the creation of an analytical approximation of objective function(s) and constrained parameters. The second step is the optimization of these approximation functions. Multi-objective optimization problem solution is based on the use of approximation functions for individual objectives and constraints.

The distinctive feature of IOSO multi-objective algorithm is an extremely low number of trial points to initialize the search procedure (typically 30 to 50 values of the objective function for the optimization problems with nearly 100 design variables). During the IOSO operation, the information concerning the behaviour of the objective function in the vicinity of the Pareto set is stored, and the response function is made more accurate only for this search area.

The main benefits of IOSO algorithm are its outstanding reliability in avoiding local minima and its computational speed.

\begin{tabular}{|c|c|c|}
\hline Objective function & Type & Efficiency of IOSO \\
\hline & Monotonious & 1.0 \\
\hline
\end{tabular}




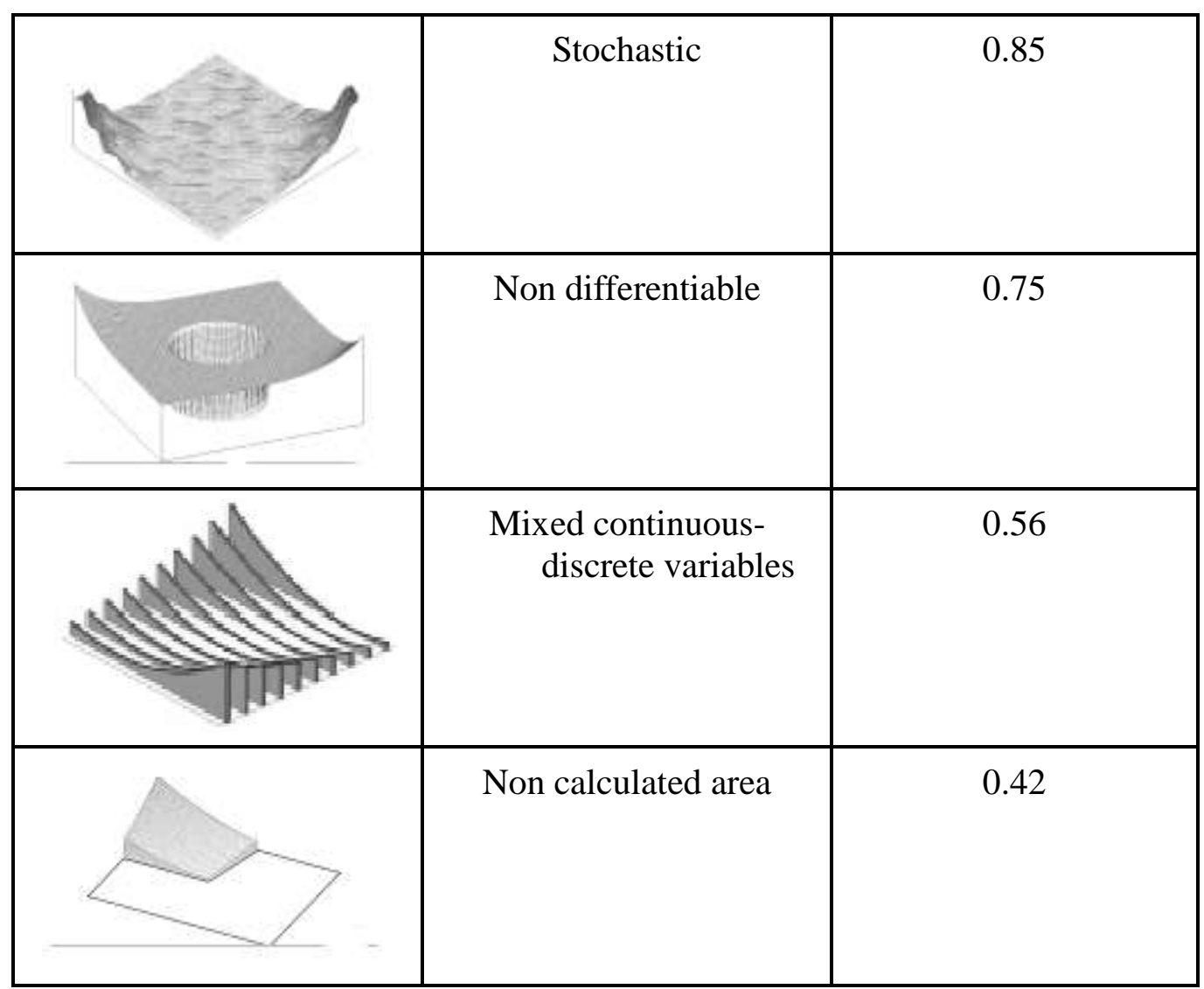

Figure 3. IOSO test on different problem-solving

The efficiency of IOSO is visible on Figure 3, considering some test examples.

Most of the real-life engineering optimization problems require simultaneous optimization of more than one objective function. In these cases, it is often impossible that the same values of design parameters will lead to the optimal values for all goals. Hence, to ensure a satisfactory design some trade-off between the objectives is necessary. For this purpose we use the multiobjective approach to optimize the overall efficiency (Egorov 1998, Egorov et al. 1997, Egorov \& Kretini 1992, Dulikravich et al. 1999).

IOSO can be easily integrated with different applications for engineering analysis both in-house and commercial, such as NASTRAN, ANSYS, StarCD, FineDesign, Fluent etc. 


\section{Novelty and distinctive features of IOSO}

- multiobjective optimization for large-dimensionality problems (up to 100 independent design variables and up to 100 constraints), which allows to reach the increase of efficiency by 2 - 7 times higher than that of middledimensionality optimization tasks (20-40 design variables);

- low expenditures for optimal solution search (reduction of the number of analysis code direct calls up to 20 times in comparison with traditional approaches and genetic algorithms (GA), depending on the complexity and dimensionality of the task);

- full automatic optimization technology algorithms with easy to use procedure of task setting;

- the possibility to solve multidisciplinary optimization problems;

- multiobjective optimization for stochastic problems (up to 100 independent design variables), having complex topology of objective and the large number of constraints. Now it is well-known that many methods are capable of solving the tasks having up to $10-20$ variables, and it is not known the analogues to IOSO optimizer that is designed for large-dimensional multiobjective tasks (100 independent design variables and 20 objectives); solving all classes of optimization problems including stochastic, multiextreme and having nondifferential peculiarities.

\section{The optimum design data and results}

The unknowns are as follows: $h, t_{1}, t_{2}, n_{x}, n_{y}$.

\section{Numerical data}

$b_{0}=8000 \mathrm{~mm}, a_{0}=24000 \mathrm{~mm}, N=3 \times 10^{7}[\mathrm{~N}], f_{y}=355 \mathrm{MPa}, E=2.1 \times 10^{5} \mathrm{MPa}$.

Ranges of variables are as follows: $t=4-40 \mathrm{~mm}, h=152.4-910.4 \mathrm{~mm}$, the maximum value of $n$ is given by the fabrication constraint (Eq. 20 or 36) 
$n_{\max }=\frac{b_{0}}{b+300}$

The $n_{\max }$ values are given in the Table 1.

Table 1. $n_{\max }$ - values for rolled I-sections - dimensions in $\mathrm{mm}$

$\begin{array}{lllllllllll}h & 353.4 & 403.2 & 454.6 & 533.1 & 607.6 & 683.5 & 762.2 & 840.7 & 910.4 & 1008.1\end{array}$

$\begin{array}{lllllllllll}b & 126.0 & 142.2 & 152.9 & 209.3 & 228.2 & 253.7 & 266.7 & 292.4 & 304.1 & 302.1\end{array}$

$\begin{array}{lllllllllll}n_{\max } & 18 & 18 & 17 & 15 & 15 & 14 & 14 & 13 & 13 & 13\end{array}$

Approximate formulae have been used to determine the UB profile dimensions in the function of the heigth $h$.

Calculation of the flange thickness $t_{f}\left(y=t_{f} ; x=h\right)$

$$
\begin{aligned}
& y=a+b x+c x^{2}+d x^{3}+e x^{4}+f x^{5}+g x^{6}+h x^{7}+i x^{8} \\
& a=-26.93815960004096 \\
& b=0.7030053163805572 \\
& c=-0.00569333794408951 \\
& d=2.383106250400329 \times 10^{-05} \\
& e=-5.605511588090933 \times 10^{-08} \\
& f=7.662794270183799 \times 10^{-11} \\
& g=-5.902409057606285 \times 10^{-14} \\
& h=2.267417890058806 \times 10^{-17} \\
& i=-2.999371273581411 \times 10^{-21}
\end{aligned}
$$

Calculation of the web thickness $t_{w}\left(y=t_{w} ; x=h\right)$

$$
\begin{aligned}
& y=a+b x+c x^{2}+d x^{3}+e x^{4}+f x^{5}+g x^{6}+h x^{7}+i x^{8} \\
& a=4.598131596507252 \\
& b=-0.1667245080692302 \\
& c=0.002662252638593643 \\
& d=-1.662919423768273 \times 10^{-05} \\
& e=5.42570607199179 \times 10^{-08} \\
& f=-1.003562930723944 \times 10^{-10} \\
& g=1.063362616433473 \times 10^{-13} \\
& h=-6.028516559742138 \times 10^{-17} \\
& i=1.419727612597333 \times 10^{-20}
\end{aligned}
$$

Calculation of the flange width $b$

$$
\begin{aligned}
& y=a+b x+c / x+d x^{2}+e / x^{2}+f x^{3}+g / x^{3}+h x^{4}+i / x^{4}+j x^{5}+k / x^{5} \\
& a=-1108926.658794802 \\
& b=2054.96457373585 \\
& c=394347552.4221416 \\
& d=-2.475920494568994 \\
& e=-91315532919.66857 \\
& f=0.001858445891156483
\end{aligned}
$$




$$
\begin{aligned}
& g=13189053888762.85 \\
& h=-7.856977790442618 \times 10^{-07} \\
& i=-1073670362507492 \\
& j=1.422535840934241 \times 10^{-10} \\
& k=3.744384150518803 \times 10^{16}
\end{aligned}
$$

\begin{tabular}{|c|c|c|c|c|c|c|c|c|}
\hline Technique & $x_{1}=t_{1}$ & $x_{2}=t_{2}$ & $x_{3}=h$ & $x_{4}=n_{x}$ & $x_{5}=n_{y}$ & Cost & Iteration & Number of \\
\hline & [mm] & {$[\mathrm{mm}]$} & {$[\mathrm{mm}]$} & & & [\$] & number & particles \\
\hline PSO & 6 & 5 & 533.1 & 2 & 12 & 46043.97 & & 50 \\
\hline$P S O$ & 8 & 5 & 454.6 & 2 & 13 & 44849.55 & & 500 \\
\hline IOSO & 11 & 4 & 454.6 & 2 & 11 & 45867.42 & 211 & \\
\hline IOSO & 8 & 4 & 454.6 & 2 & 14 & 43769.13 & 522 & \\
\hline
\end{tabular}

Table 2 shows the discrete optima using PSO and IOSO techniques. The number of stiffeners in $x$ direction is relatively small.

Table 2. Optimum values for the cellular plate - dimensions in mm, cost in USD

The discrete values are found finding the continuous ones. At IOSO a parametric study is needed to do this. At PSO there is a built in calculation for this. The number of stiffeners and height of stiffeners have no conflict as it is given in Table 1.

\section{Conclusions}

Cellular plates are constructed from two base plates and an orthogonal grid of stiffeners welded between them. Such plates have a large torsional stiffness, which makes the plate very stiff and economic.

In the case of uniaxial compression the overall buckling constraint is derived from the Huber's equation for orthotropic plates. The local buckling of plate elements is considered by effective widths. The fabrication constraint expresses that the 
distance between the stiffeners should be sufficient for welding the stiffeners to the upper face plate.

The unknowns are as follows: upper and lower cover plate thickness, height of the halved rolled I-stiffeners, number of stiffeners in $x$ - and $y$-directions. The cost function contains the cost of material, assembly and welding and is formulated according to the fabrication sequence.

The Particle Swarm Optimization and the IOSO techniques are used to find the optimum. The two optimizers give nearly the same solution. Both of them are very robust techniques.

Acknowledgements

The research was supported by the Hungarian Scientific Research Fund OTKA T 75678 and by the TÁMOP 4.2.1.B-10/2/KONV-2010-0001 entitled Increasing the quality of higher education through the development of research - development and innovation program at the University of Miskolc.

\section{References}

Birchfield J.R. (1981) Welded machines thrive on tough mining. Welding Design and Fabrication 1981; 54 No.4, 47-54.

Det Norske Veritas (DNV) (1995) Buckling strength analysis, Classification Notes No.30.1. Høvik, Norway.

Dulikravich,G.S., Martin,T.J., Dennis,B.H. and Foster,N.F. (1999) Multidisciplinary Hybrid Constrained GA Optimization," Chapter 12 in EUROGEN'99 - Evolutionary Algorithms in Engineering and Computer Science: Recent Advances and Industrial Applications, ed. K. Miettinen, M.M. Makela, P. Neittaanmaki and J. Periaux (John Wiley \& Sons, Ltd.), Jyvaskyla, Finland, May 30 - June 3 (1999), 231-260.

Eurocode 3 (2006) Design of steel structures. Part 1-5: Plated structural elements.

Egorov,I.N. (1998) Indirect Optimization Method on the Basis of Self-Organization,"Proceedings of Optimization Techniques and Applications (ICOTA'98),_2, 683-691, Curtin University of Technology, Perth, Australia.

Egorov,I.N, Kretinin,G.V. and Leshchenko,I.A. (1997) Multicriteria Optimization of Time Control Laws of Short Take-Off and Vertical Landing Aircraft Power Plant, ASME paper 97-GT-263

Egorov,I.N. and Kretinin,G.V. (1992) Multicriterion Stochastic Optimization of Axial Compressor, Proceedings of ASME COGEN-TURBO-VI, Houston, Texas

Evans,H.R, Shanmugam,N.E. (1984) Simplified analysis for cellular structures. J. Struct. Eng ASCE 1984; 110, 531-543.

Farkas,J. (1985) Discussion to the paper of Evans R, Shanmugam NE. Simplified analysis for cellular structures. J. Struct. Eng ASCE, 1985; 11: No.10, 2269-71.

Farkas,J., Jármai,K. (1997) Analysis and optimum design of metal structures. Balkema, Rotterdam-Brookfield

Farkas,J., Jármai,K. (2003) Economic design of metal structures. Millpress, Rotterdam

Farkas,J., Jármai,K. (2006) Optimum design and cost comparison of a welded plate stiffened on one side and a cellular plate both loaded by uniaxial compression. Welding in the World, 2006; 50: No.3-4, 45-51.

Farkas,J., Jármai,K. (2008) Design and optimization of metal structures. Horwood, Chichester, UK 
Fourie,P.C. \& Groenwold,A.A. (2000) Particle swarm in size and shape optimisation, International Workshop on Multidisciplinary Design Optimization, 7-10, Aug. 2000, Pretoria, South Africa, Proceedings 97-106.

Haroutel,J. (1982) Soudage laser de structures sandwich métalliques du type Norsial. Soudage et Techniques Connexes 1982; Jan-Fevr., 25-31.

Jármai,K., Farkas,J. (1999) Cost calculation and optimization of welded steel structures. Journal of Constructional Steel Research 1999; 50 115-135

Kennedy,J. \& Eberhart,R.C. (1995) Particle swarm optimization. Proc. IEEE Int'l Conf. on Neural Networks, IV, 1942-1948. IEEE service center, Piscataway, NJ, 1995. 1942-1948

Krack, M., Secanell, M. and Mertiny P. (2011) Cost optimization of a hybrid composite flywheel rotor with a split-type hub using combined analytical/numerical models, Structural and Multidisciplinary Optimization Volume 44, Number 1, 57-73, DOI: 10.1007/s00158-010-0573Z

Kravanja,S., Zula,T.,Klansek,U. (2008) The MINLP approach to cost optimization of structures. in Design, fabrication and economy of welded structures: International Conference Proceedings, Miskolc, Hungary, 2008, 24 - 26 April, Horwood Publishers, Chichester, UK, 2008. pp. 89-96.

Pavlovcic,L.,Krajnc,A.,Beg,D. (2004) Cost function analysis in the structural optimization of steel frames, Structural and Multidisciplinary Optimization, Vol. 28. No. 4, pp. 286-295.

Pettersen,E. (1979) Analysis and design of cellular structures. University of Trondheim, Norwegian Institute of Technology.

Shanmugam,N.E, Evans,H.R. (1984) A grillage analysis of the nonlinear and ultimate load behavior of cellular structures under bending loads. Proc. Inst. Civ. Eng. Part 2. 1984; 71, 705719

Shanmugam,N.E, Balendra,T. (1986) Free vibration of thin-walled multi-cell structures. Thinwalled Struct. 1986; 4, 467-483

Sahmel,P. (1978) Statische und konstruktive Probleme bei Hilfsvorrichtungen zum Transport schwerer Behälter. Fördern und Heben 1978; 28, 844-7.

Sarma,K.C., Adeli,H. (2002) Life -cycle cost optimization of steel structures, Wiley Publishers, DOI: $10.1002 / \mathrm{nme} .549$

Williams,D.G. (1969) Analysis of doubly plated grillage under inplane and normal loading. $\mathrm{PhD}$ Thesis. Imperial College, London

Xiong, F., Chen, W., Xiong, Y. and Yang, S. (2011) Weighted stochastic response surface method considering sample weights, Structural and Multidisciplinary Optimization Volume 43, Number 6, 837-849, DOI: 10.1007/s00158-011-0621-3 